\title{
Vasorelaxant Activity of Sappan Lignum Constituents and Extracts on Rat Aorta and Mesenteric Artery
}

\author{
Yohei Sasaki, ${ }^{a}$ Maemi Suzuki, ${ }^{a}$ Takayuki Matsumoto, ${ }^{b}$ Tomokazu Hosokawa, ${ }^{a}$ Tsuneo Kobayashi, ${ }^{b}$ \\ Katsuo Kamata, ${ }^{b}$ and Seiji NaGUMO ${ }^{*, a}$ \\ ${ }^{a}$ Department of Medicinal Plant Sciences, Hoshi University; and ${ }^{b}$ Department of Physiology and Morphology, Institute of \\ Medicinal Chemistry, Hoshi University; 2-4-41 Ebara, Shinagawa, Tokyo 142-8501, Japan. \\ Received February 13, 2010; accepted June 14, 2010; published online June 16, 2010
}

\begin{abstract}
We investigated the vasorelaxant activity of the methanolic extracts of Sappan Lignum (CSE) and its constituents, brazilin, sappanchalcone, and protosappanins $\mathrm{A}-\mathrm{E}$, on rat aorta and mesenteric artery. By comparing the vasorelaxant activity of CSE and brazilin on both blood vessels, we found that CSE contained active constituents other than brazilin. When added to brazilin, sappanchalcone and protosappanin D showed vasorelaxant activity on both blood vessels precontracted with phenylephrine. We clarified that the vasorelaxant activity of brazilin was endothelium-independent, while that of sappanchalcone was endothelium-dependent, on both blood vessels. On the other hand, the vasorelaxant activity of protosappanin $D$ was independent of the endothelium of the aorta and dependent on the endothelium of the mesenteric artery. Experiments on sappanchalcone and protosappanin $\mathrm{D}$ using $N^{\mathrm{G}}$-nitro-L-arginine and indomethacin revealed the involvement of nitric oxide and prostaglandin as endothelium-derived relaxation factors (EDRFs). The anti-oketsu effect of Sappan Lignum might be attributable to the interaction of those compounds. We could partly evaluate the anti-oketsu activity of Sappan Lignum using both the aorta and the mesenteric artery. Through this study, we showed the importance of comparing the effects on the aorta and the mesenteric artery as we found that natural compounds showed different mechanisms of action on the two blood vessels.
\end{abstract}

Key words Caesalpinia sappan; Sappan Lignum; vasorelaxant activity; aorta; mesenteric artery

Sappan Lignum is derived from the heartwood of Caesalpinia sappan L. (Leguminosae) and used as dyestuff or herbal medicine. It is used as an anti-inflammatory agent ${ }^{1)}$ or an anti-oketsu agent ${ }^{2}$ (the oketsu syndrome is caused by the obstruction of blood circulation) in Japan.

Recently, the anti-influenza viral activity, ${ }^{3)}$ the anti-allergic activity, ${ }^{4)}$ and the neuroprotective activity ${ }^{5}$ of Sappan Lignum or brazilin, the major constituent of Sappan Lignum, were reported. Although other compounds, including sappanchalcone and protosappanins $\mathrm{A}-\mathrm{E}$, are present in Sappan Lignum, there are few reports of their pharmacological activities.

As regards the traditional use of Sappan Lignum, we reported the anti-inflammatory activities of the seven compounds examined in an in vitro assay. ${ }^{6}$. However, there are few reports of the anti-oketsu effects of the constituents of Sappan Lignum. There are also few reports of the effect of brazilin on vasomotion. ${ }^{7,8)}$

To evaluate the anti-oketsu effects of herbal medicines, the vasorelaxant activity of herbal medicines on isolated rat aorta is commonly examined. However, the use of only aorta as the test blood vessel is inadequate because the obstruction of blood circulation during oketsu is thought to occur in peripheral blood vessels. Generally, it is known that the contribution of endothelium-derived relaxation factors (EDRFs), such as nitric oxide (NO), prostaglandin $\mathrm{I}_{2}\left(\mathrm{PGI}_{2}\right)$ or endotheliumderived hyperpolarizing factor (EDHF), to endotheliumdependent relaxation varies as a function of blood vessel size. ${ }^{9-11)}$ Although NO, an EDRF, is the most prominent vasorelaxant in the aorta, the contribution of NO becomes less in small blood vessels. Therefore, compounds that show vasorelaxant activity on the aorta do not always show the same activity on small blood vessels.
In this study, we evaluated the vasorelaxant activity of the methanolic extracts of Sappan Lignum (CSE) and its constituents, brazilin, sappanchalcone, and protosappanins AE, on rat aorta and mesenteric artery. To our knowledge, our work is the first to compare the effects of natural compounds on both blood vessels.

\section{MATERIALS AND METHODS}

Materials Brazilin (1, 5.1\% from CSE), sappanchalcone ${ }^{12)}$ (2, $0.38 \%)$, protosappanin $\mathrm{A}^{13)}(3,0.15 \%)$, protosappanin $\mathrm{B}^{14)}(\mathbf{4}, 0.80 \%)$, protosappanin $\mathrm{C}^{15)}(\mathbf{5}, 0.48 \%)$, protosappanin $\mathrm{D}^{16)}(\mathbf{6}, 0.15 \%$, isomeric mixture), and protosappanin $\mathrm{E}^{17)}(7,0.32 \%$, isomeric mixture of E-1, E-2) were isolated from Sappan Lignum (Fig. 1) purchased from Uchida Wakanyaku Co., Ltd. (Lot. No. 313116, December 27, 2006).

Chemical Reagents Phenylephrine, $N^{\mathrm{G}}$-nitro-L-arginine (L-NNA), indomethacin, and acetylcholine chloride were purchased from Sigma Chemicals (St. Louis, MO, U.S.A.). All the drugs except indomethacin were dissolved in saline. Indomethacin was first dissolved in a small amount of $0.1 \mathrm{M}$ $\mathrm{Na}_{2} \mathrm{CO}_{3}$ solution and then made up to the final volume with distilled water.

Animals Male Wistar rats weighing 350-450g were purchased from Tokyo Laboratory Animals Science Co., Ltd. (Tokyo, Japan). This study was carried out in accordance with the guidelines set by the Committee on the Care and Use of Laboratory Animals of Hoshi University.

Measurement of Isometric Force ${ }^{18)}$ Male Wistar rats were anesthetized with diethyl ether and then euthanized by decapitation. The superior mesenteric artery and the thoracic aorta were rapidly removed and immersed in oxygenated, modified Krebs-Henseleit solution (KHS). This solution 

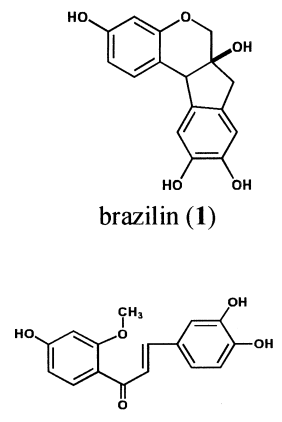

sappanchalcone (2)

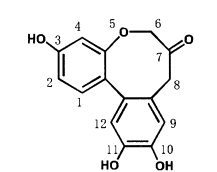

protosappanin A (3)
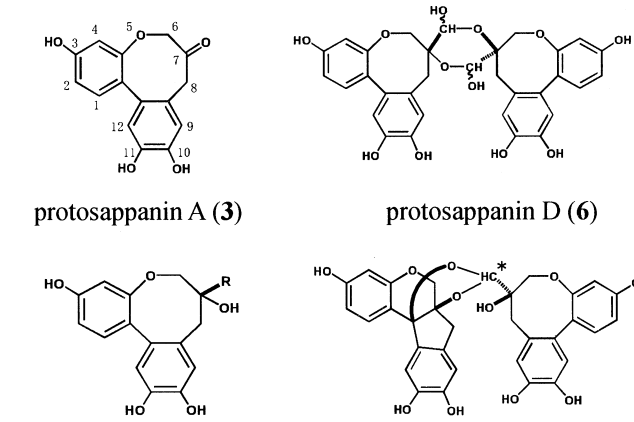

$\mathbf{R}=\mathrm{CH}_{2} \mathrm{OH}$ : protosappanin $\mathrm{B}(\mathbf{4})$

$\mathbf{R}=\mathbf{C H O}$ : protosappanin $\mathrm{C}(\mathbf{5})$

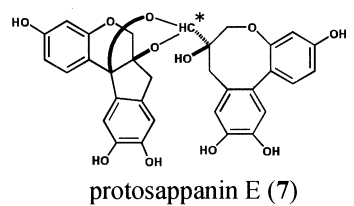

Fig. 1. Structures of Compounds Used in This Study

consisted of (in mM) $118.0 \mathrm{NaCl}, 4.7 \mathrm{KCl}, 1.8 \mathrm{CaCl}_{2}, 1.2$ $\mathrm{MgSO}_{4}, 1.2 \mathrm{NaH}_{2} \mathrm{PO}_{4}, 25.0 \mathrm{NaHCO}_{3}$, and 11.1 glucose. The artery was carefully cleaned of fat and connective tissues, and ring preparations of $3 \mathrm{~mm}$ (aorta) or $2 \mathrm{~mm}$ (mesenteric artery) length were suspended by a pair of stainless steel pins in a well-oxygenated $\left(95 \% \mathrm{O}_{2}-5 \% \mathrm{CO}_{2}\right)$ bath containing 5.0 $\mathrm{ml}$ of KHS at $37^{\circ} \mathrm{C}$. The rings were stretched until an optimal resting tension of $1.0 \mathrm{~g}$ was loaded. Then, the rings were equilibrated for at least $60 \mathrm{~min}$. Force generation was monitored by means of an isometric transducer (TB-611T, Nihon Kohden, Tokyo, Japan).

Before the experiments, each ring was precontracted with phenylephrine $\left(10^{-5} \mathrm{M}\right)$ and then relaxed with acetylcholine $\left(10^{-5} \mathrm{M}\right)$. After washing fully, each ring was precontracted with phenylephrine $\left(10^{-6} \mathrm{M}\right)$ again. After the phenylephrineinduced contraction reached a plateau, acetylcholine $\left(10^{-9}-10^{-5} \mathrm{M}\right)$ was added in a cumulative manner. The existence of the endothelium was confirmed from the percentage relaxation. The relaxation with acetylcholine was not observed the denuded ring.

In the experiments, each ring was precontracted with phenylephrine $\left(10^{-6} \mathrm{M}\right)$. Then, CSE was added at concentrations ranging from $10^{-6}$ to $3 \times 10^{-4} \mathrm{~g} / \mathrm{ml}$, or the compounds were added at concentrations ranging from $10^{-7}$ to $3 \times 10^{-5} \mathrm{M}$ cumulatively or at the concentration of $3 \times 10^{-5} \mathrm{M}$, when the phenylephrine-induced contraction reached a plateau.

Relaxation was expressed as the percentage decrease in the maximal tension attained after pretreatment with phenylephrine. To investigate the involvement of $\mathrm{NO}$ or prostaglandin, ring preparations with the endothelium were exposed to $10^{-4} \mathrm{M}$ L-NNA, an inhibitor of NO synthase (NOS), and/or $10^{-5} \mathrm{M}$ indomethacin, an inhibitor of cyclooxygenase (COX), for 30 min before precontraction.

Statistical Analysis All data are expressed as means and S.E.M. Statistical comparisons were made by Student's $t$-test for unpaired data and Tukey's test for multiple comparisons. The differences were considered significant at $p<0.05$.

\section{RESULTS}

Vasorelaxant Activity of CSE and Compound 1 on Aorta and Mesenteric Artery First, we tried to determine whether brazilin (compound $\mathbf{1}$; main constituent of CSE) is the only compound that shows vasorelaxant activity on blood vessels or not. CSE was added cumulatively to the bath and relaxation was induced in each blood vessel at the concentra- tion of $10^{-5} \mathrm{~g} / \mathrm{ml}$ (Fig. 2). When CSE was added at the concentrations of $10^{-5}, 3 \times 10^{-5}$, and $10^{-4} \mathrm{~g} / \mathrm{ml}$, the percentage relaxation of the aorta was $38 \%, 85 \%$, and $92 \%$, respectively, and that of the mesenteric artery was $51 \%, 94 \%$, and $93 \%$, respectively. On the other hand, compound $\mathbf{1}$ at $10^{-5}$ and $3 \times 10^{-5} \mathrm{M}$ induced $13 \%$ and $100 \%$ relaxation of the aorta, and $31 \%$ and $99 \%$ relaxation of the mesenteric artery, respectively. In contrast to the vasorelaxant papaverine that induced the relaxation immediately, both CSE and compound 1 induced the relaxation of each blood vessel slowly. We did not observe any differences in vasorelaxant activity between CSE and compound $\mathbf{1}$ on both blood vessels. Compound $\mathbf{1}$ is brazilin $\left(\mathrm{C}_{16} \mathrm{H}_{14} \mathrm{O}_{5}\right.$; molecular weight: 286.28$)$ and is present in CSE at approximately $5 \%$. Thus, the concentration of compound 1 at $3 \times 10^{-5} \mathrm{M}$ is equivalent to approximately $1.7 \times 10^{-4} \mathrm{~g} / \mathrm{ml}$ of CSE. By comparing the percentage relaxation of the aorta and the mesenteric artery around that concentration, we found that CSE contained active constituents other than compound $\mathbf{1}$ (brazilin).

Vasorelaxant Activity of Seven Compounds on Aorta and Mesenteric Artery Next, the seven compounds were added cumulatively to the bath. All the compounds at the concentration of $1.0 \times 10^{-5} \mathrm{M}$ had weak vasorelaxant activity on both blood vessels. The percentage relaxation at $1.0 \times$ $10^{-5} \mathrm{M}$ and $3.0 \times 10^{-5} \mathrm{M}$ is shown in Fig. 3. In the case of compounds $\mathbf{1}, \mathbf{2}$, and $\mathbf{6}$, the differences in vasorelaxant activity between $1.0 \times 10^{-5} \mathrm{M}$ and $3.0 \times 10^{-5} \mathrm{M}$ were more than $60 \%$ in both blood vessels. These results indicate that the vasorelaxant activity of the compounds appeared at the concentration of $3.0 \times 10^{-5} \mathrm{M}$. Then, we conducted the singledose administration assay in the next experiment using compounds $\mathbf{1}, \mathbf{2}$, and $\mathbf{6}$.

The vasorelaxant activities of compounds $\mathbf{3}, \mathbf{4}, \mathbf{5}$, and $\mathbf{7}$ on both blood vessels were less than $60 \%$ and the activities tended to be stronger on the mesenteric artery than on the aorta. The percentage relaxation at $3.0 \times 10^{-5} \mathrm{M}$ was $4 \%$ (compound 3), 28\% (4), 27\% (5), and 51\% (7) for the aorta, while it was $23 \%(3), 37 \%(4), 37 \%(5)$, and $53 \%(7)$ for the mesenteric artery.

Effects of Compounds 1, 2, and 6 on EndotheliumDenuded Aorta and Mesenteric Artery For compounds 1, $\mathbf{2}$, and $\mathbf{6}$, the single-dose administration assay was conducted on the aorta and the mesenteric artery with and without endothelium, and the time course of relaxation was observed (Fig. 4). The three compounds showed vasorelaxant activity on both intact blood vessels compared to the vehicle $(0.01 \%$ 


\section{A. Aorta (intact)}

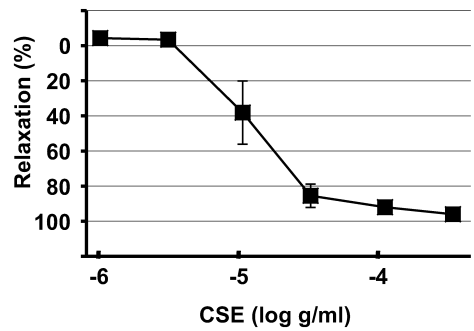

B. Mesenteric artery (intact)

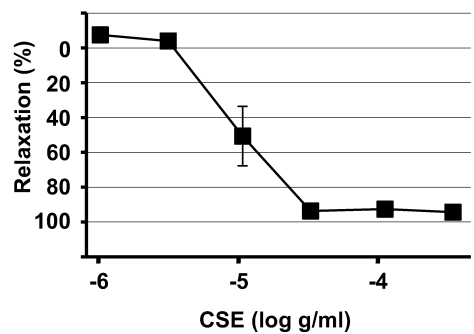

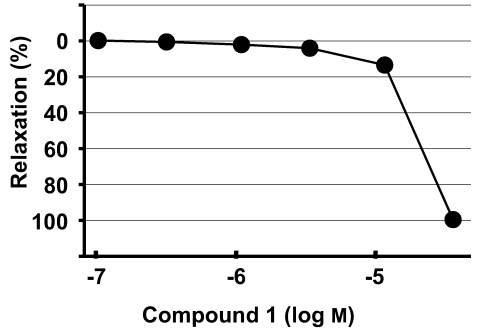

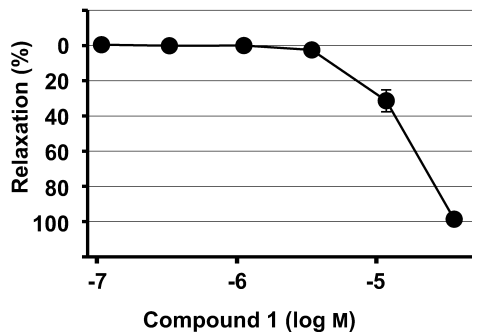

Fig. 2. Concentration-Response Curves for CSE- or Compound-1-Induced Relaxation of Intact Aortic Rings (A) and Intact Mesenteric Artery Rings (B)

The rings were pretreated with phenylephrine $\left(10^{-6} \mathrm{M}\right)$. CSE was cumulatively added at concentrations ranging from $10^{-6} \mathrm{M}$ to $3 \times 10^{-4} \mathrm{~g} / \mathrm{ml}$, and compound $\mathbf{1}$ was cumulatively added at concentrations ranging from $10^{-7}$ to $3 \times 10^{-5} \mathrm{M}$. Values are expressed as the percentage decrease in the maximal tension attained after pretreatment with phenylephrine. Results are means \pm S.E.M. of four to five experiments.

\section{A. Aorta (intact)}

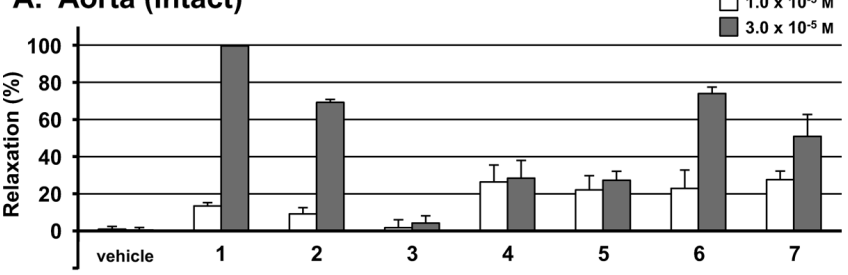

B. Mesenteric artery (intact)

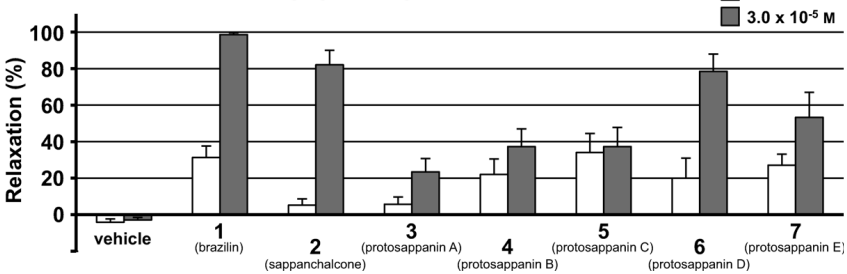

Fig. 3. Vasorelaxant Activity of Seven Isolated Compounds on Intact Aortic Rings (A) and Intact Mesenteric Artery Rings (B) at Concentrations of $10^{-5} \mathrm{M}$ and $3 \times 10^{-5} \mathrm{M}$

The rings were pretreated with phenylephrine $\left(10^{-6} \mathrm{M}\right)$ and the compounds were cumulatively added at concentrations ranging from $10^{-7}$ to $3 \times 10^{-5} \mathrm{M}$. The values at concentrations of $10^{-5} \mathrm{M}$ and $3 \times 10^{-5} \mathrm{M}$ are shown in the upper bar graph. Values are expressed as the percentage decrease in the maximal tension attained after pretreatment with phenylephrine. Results are means \pm S.E.M. of four to five experiments.

methyl sulfoxide (DMSO)). At $60 \mathrm{~min}$, the percentage relaxation of the intact aorta was $94 \%, 73 \%$, and $63 \%$ on treatment with compounds 1, 2, and 6, respectively (Fig. $4 \mathrm{~A}_{1}$ ), and that of the mesenteric artery was $97 \%, 100 \%$, and $79 \%$, respectively (Fig. $4 \mathrm{~B}_{1}$ ). In the experiments of denuded blood vessels, the vasorelaxant activity of compound $\mathbf{1}$ on intact and denuded blood vessels showed no significant difference. The percentage relaxation of the denuded aorta and the denuded mesenteric artery was $96 \%$ and $94 \%$ on treatment with compound 1 , respectively (Figs. $4 \mathrm{~A}_{2}, \mathrm{~B}_{2}$ ). In contrast, the vasorelaxant activity of compound $\mathbf{2}$ on the denuded blood vessels almost disappeared; the percentage relaxation was 5\% (aorta) and 9\% (mesenteric artery) at $60 \mathrm{~min}$. The percentage relaxation of the denuded blood vessels was diminished to $61 \%$ (aorta) and $18 \%$ (mesenteric artery) at $60 \mathrm{~min}$ on treatment with compound 6. Together, these results indicate that 1 ) compound $\mathbf{1}$ induced relaxation independently of the endothelium of both blood vessels; 2) compound $\mathbf{2}$ induced relaxation via the endothelium of both blood vessels; and 3) compound $\mathbf{6}$ induced relaxation independently of the endothelium of the aorta and via the endothelium of the mesenteric artery.

Effects of Compounds 1, 2, and 6 on Intact Aorta and Mesenteric Artery Pretreated with L-NNA and/ or Indomethacin To check the mechanisms involved in the vasorelaxant activities of the compounds, intact blood vessels were pretreated with L-NNA (NOS inhibitor) and/or indomethacin (COX inhibitor).

Compound 1, which was revealed to induce relaxation without involvement of the endothelium in the experiments that used denuded blood vessels, did not show differences in vasorelaxant activity with and without L-NNA pretreatment (Fig. 5A). Meanwhile, the vasorelaxant activity of compound 2 was largely suppressed by pretreatment with L-NNA; percentage relaxation was reduced from $73 \%$ (aorta without LNNA) to $12 \%$ (aorta with L-NNA), and from 100\% (mesenteric artery without L-NNA) to $31 \%$ (mesenteric artery with L-NNA) (Fig. 5B). Moreover, the vasorelaxant activity of compound 2 was affected by prostaglandin; percentage relaxation was reduced from $73 \%$ (aorta without indomethacin) to $38 \%$ (aorta with indomethacin), and from 100\% (mesenteric artery without indomethacin) to $42 \%$ (mesenteric artery with indomethacin). As regards compound 6, although its vasorelaxant activity on the mesenteric artery was strongly inhibited by L-NNA and/or indomethacin, we could not find complete inhibition of its activity on the aorta by indomethacin 

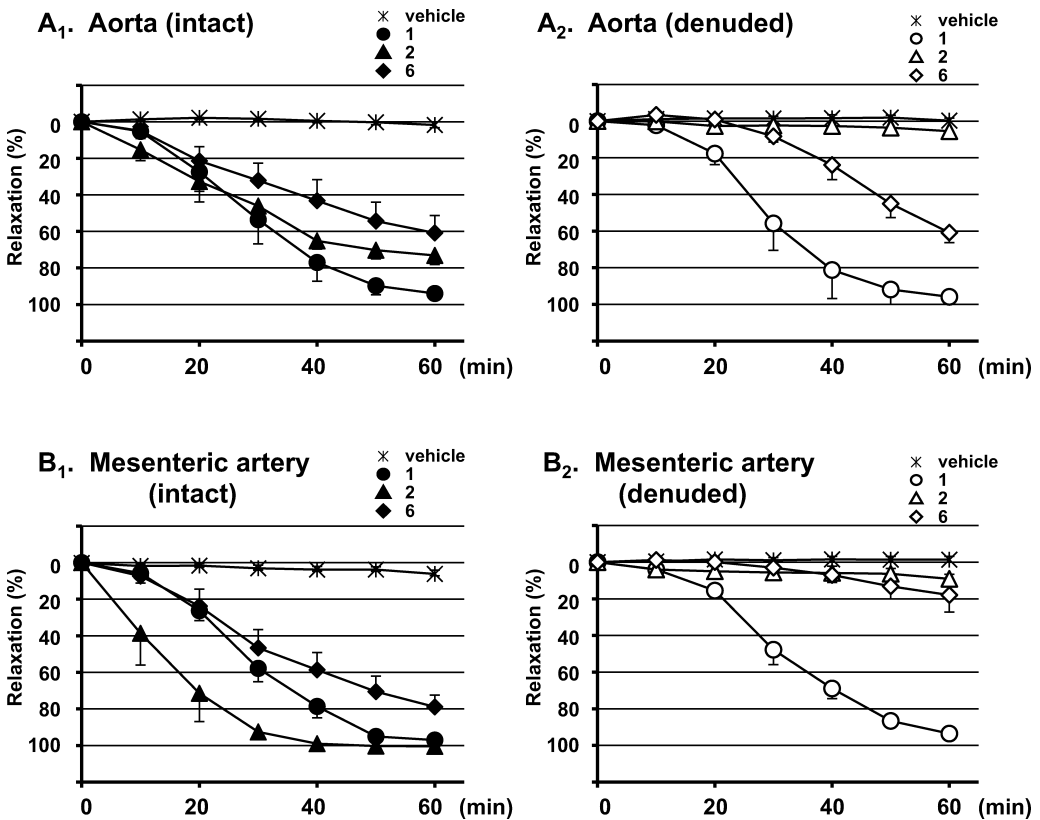

Fig. 4. Time Course of Relaxation of Intact $\left(\mathrm{A}_{1}\right)$ and Denuded $\left(\mathrm{A}_{2}\right)$ Aortic Rings and Intact $\left(\mathrm{B}_{1}\right)$ and Denuded $\left(\mathrm{B}_{2}\right)$ Mesenteric Artery Rings Induced by Compounds $\mathbf{1}, \mathbf{2}$, and $\mathbf{6}$

The intact or denuded rings were pretreated with phenylephrine $\left(10^{-6} \mathrm{M}\right)$. The compounds were added at the concentration of $3 \times 10^{-5} \mathrm{M}$. Values are expressed as the percentage decrease in the maximal tension attained after pretreatment with phenylephrine. Results are means \pm S.E.M. of four to seven experiments.

\section{Aorta}

Comp. $1\left(3.0 \times 10^{-5} \mathrm{M}\right)$

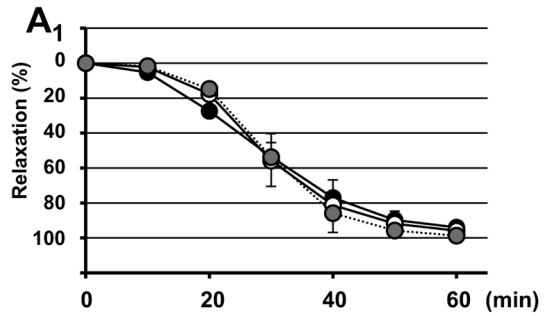

Comp. $2\left(3.0 \times 10^{-5} \mathrm{M}\right)$
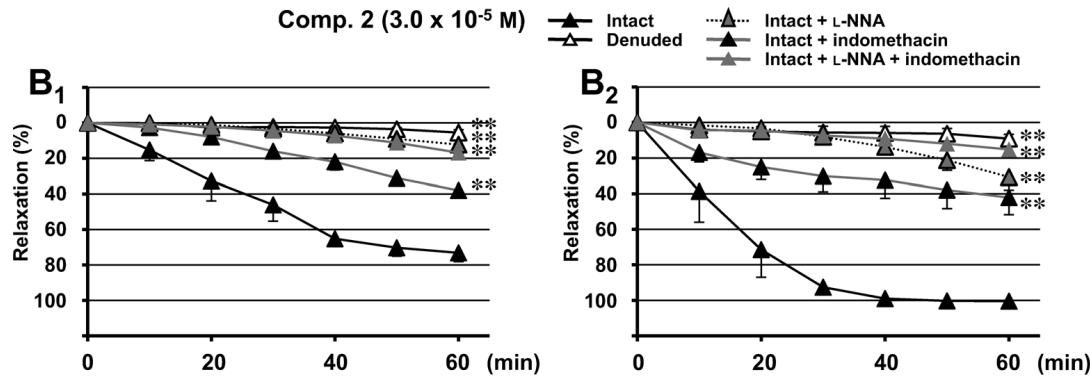

\section{Mesenteric artery}
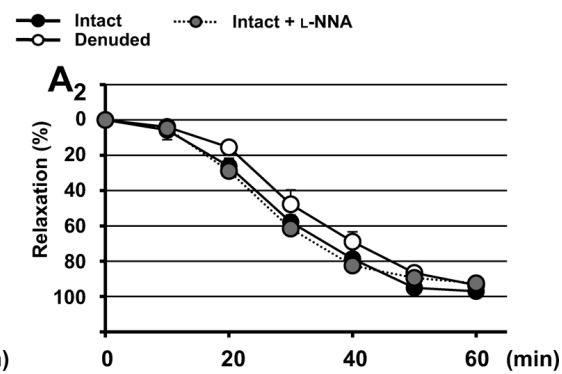
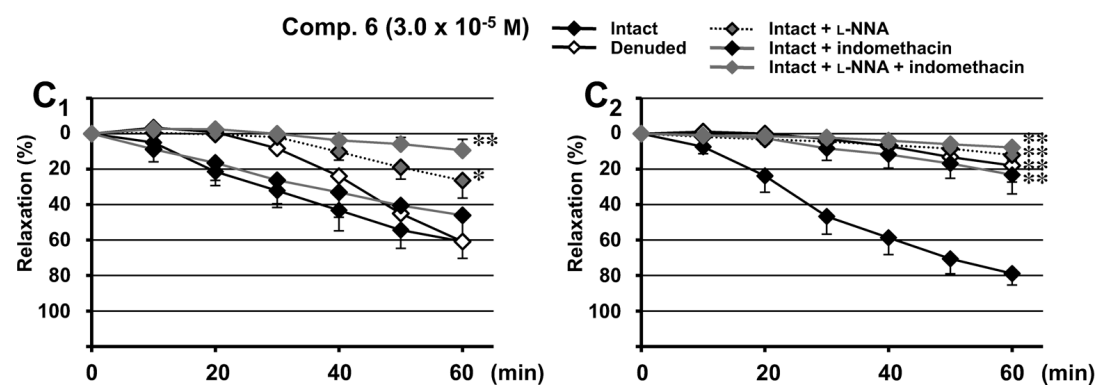

Fig. 5. Time Course of Relaxation of Aortic Rings $\left(A_{1}, B_{1}, C_{1}\right)$ and Mesenteric Artery Rings $\left(A_{2}, B_{2}, C_{2}\right)$ Induced by Compounds $\mathbf{1}, \mathbf{2}$, and $\mathbf{6}$

The intact rings, denuded rings, or intact rings exposed to L-NNA and/or indomethacin were pretreated with phenylephrine $\left(10^{-6} \mathrm{M}\right)$. The compounds were added at the concentration of $3 \times 10^{-5} \mathrm{M}$. Values are expressed as the percentage decrease in the maximal tension attained after pretreatment with phenylephrine. Results are means \pm S.E.M. of four to nine experiments. $* p<0.05$, $* * p<0.01, v s$. denuded rings. 


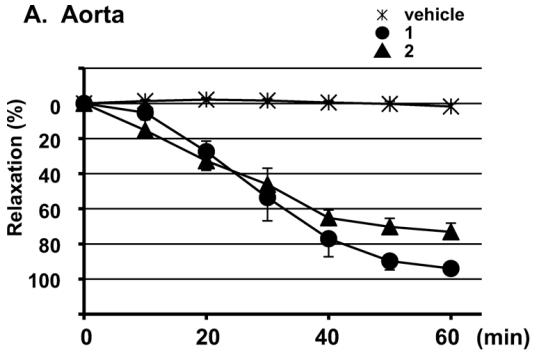

C. Aorta

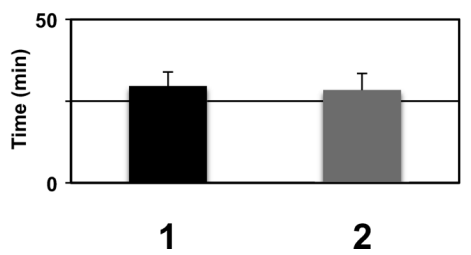

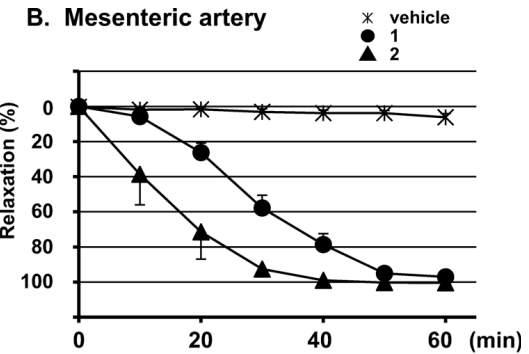

D. Mesenteric artery

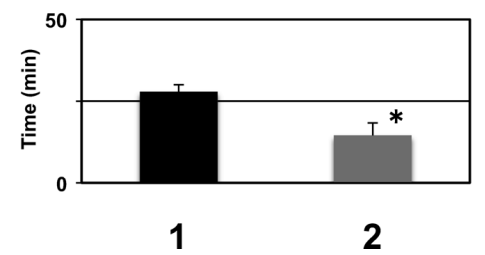

Fig. 6. Time Course of Relaxation of Aortic Rings (A) and Mesenteric Artery Rings (B), and the Time When Vasorelaxant Activity on Aortic Rings (C) and Mesenteric Artery Rings (D) Induced by Compounds 1 and 2 was $50 \%$

$(A, B)$ The intact rings were pretreated with phenylephrine $\left(10^{-6} \mathrm{M}\right)$. The compounds were added at the concentration of $3 \times 10^{-5} \mathrm{M}$. $(\mathrm{C}, \mathrm{D}) \mathrm{The}$ time when vasorelaxant activity was $50 \%$, calculated from the time course graphs. Values are expressed as the percentage decrease in the maximal tension attained after pretreatment with phenylephrine. Results are means \pm S.E.M. of four to six experiments. $* p<0.05$, $v$ s. compound $\mathbf{1}$ on mesenteric artery.

(Fig. 5C). The influence of prostaglandin on the compound 6 induced relaxation was significantly greater on the mesenteric artery than the aorta.

Comparison of Time-Response Curve between Aorta and Mesenteric Artery We compared the time course of relaxation induced by compounds $\mathbf{1}$ and $\mathbf{2}$ because we found that compound $\mathbf{2}$ showed rapid vasorelaxant activity on the mesenteric artery (Figs. 6A, B). The time when the percentage relaxation induced by the two compounds on the aorta was $50 \%$ was almost the same, although a shorter time was noted for compound $\mathbf{2}$ than compound $\mathbf{1}$ in the case of the mesenteric artery (Figs. 6C,D). The percentage relaxation induced by both compounds finally reached approximately $100 \%$ at $60 \mathrm{~min}$. We found that the compounds showed different effects on the aorta and the mesenteric artery.

\section{DISCUSSION}

We clarified that compounds $\mathbf{1}, \mathbf{2}$, and $\mathbf{6}$ possessed vasorelaxant activity on rat aorta and mesenteric artery and that their relaxation mechanisms were different. The vasorelaxant activity of compound $\mathbf{1}$ on both blood vessels was endothelium-independent.

Xie et al. reported the vasorelaxant activity of brazilin on rat aorta precontracted with phenylephrine $\left(10^{-7} \mathrm{M}\right) .^{7)}$ They concluded that brazilin at $3 \times 10^{-5} \mathrm{M}$ was NO-dependent and that at $10^{-4} \mathrm{M}$ was NO-independent. On the other hand, $\mathrm{Hu}$ et al. observed that brazilin at $3 \times 10^{-4} \mathrm{M}$ and $1 \times 10^{-3} \mathrm{M}$ showed endothelium-dependent vasorelaxation through $\mathrm{NO}$ on rat aorta precontracted with phenylephrine $\left(3 \times 10^{-6} \mathrm{M}\right){ }^{8)}$ The discrepancy between those studies may be partly explained by the differences in the purity of brazilin.

The vasorelaxant activity of compound $\mathbf{2}$ on both blood vessels was endothelium-dependent. Vasorelaxant activity was suppressed by NOS inhibitor and partly suppressed by COX inhibitor. The vasorelaxant activity and the mechanism of action of compound $\mathbf{6}$ were different between the aorta and the mesenteric artery. The vasorelaxant activity of com- pound $\mathbf{6}$ was independent of the endothelium in the aorta and dependent on the endothelium in the mesenteric artery. We could not clarify the detailed mechanism of the endotheliumdependent vasorelaxant activity of compound $\mathbf{2}$ on the aorta and the mesenteric artery and that of compound $\mathbf{6}$ on the mesenteric artery. However, it was suggested that those compounds inhibited NOS and COX through different mechanisms because the total percentage reduction of vasorelaxant activity by NOS inhibitor and COX inhibitor exceeded $100 \%$.

The relaxation induced by compounds 1, 2, and $\mathbf{6}$ was slow compared with that by papaverine. Moreover, the time required to induce $50 \%$ vasorelaxant activity on the mesenteric artery by compound $\mathbf{2}$ was shorter than the time required by compound $\mathbf{1}$, while the time required to induce the activity on the aorta was not different between compounds $\mathbf{1}$ and 2 . The long time required for vasorelaxation may be due to the solubility of the compounds originating from CSE in the buffer. Better results could be obtained if an in vivo study were performed.

In terms of chemical structure, only compound $\mathbf{6}$ is pharmacologically active among the protosappanins, similar to compounds $\mathbf{1}$ and 2 . Compound $\mathbf{6}$ is a dimer of two molecules of compound $\mathbf{5}$. Compound $\mathbf{5}$ does not show strong vasorelaxant activity. On the other hand, compound $\mathbf{1}$ is pharmacologically active whereas compound 7, the dimer of compounds $\mathbf{1}$ and $\mathbf{5}$, is not. Interestingly, our previous antiinflammation studies also concluded that compounds $\mathbf{1}, \mathbf{2}$, and $\mathbf{6}$ are pharmacologically active compounds. ${ }^{6,19)}$ Although it is unknown whether anti-inflammatory activity and vasorelaxant activity share the same pharmacological mechanisms, those compounds are suggested to be effective against oketsu, because oketsu is thought to be cured by reducing inflammation and improving blood circulation.

In the past, the evaluation of anti-oketsu herbal medicine was performed using rat aorta. It was considered to be important that the aorta was relaxed by the herbal medicine through NO from the endothelium because NO is the main 
EDRF in the aorta. At present, it is known that the contribution of NO in the mesenteric artery is approximately 17 to $20 \%$ while that in the aorta is approximately $50 \%$. As the oketsu syndrome is thought to be prominent in peripheral tissues, examinations that use only the aorta are insufficient. Although we know of a few studies of the vasorelaxant activity of natural compounds using rat mesenteric artery, ${ }^{20,21)}$ those studies did not conduct the evaluation from the viewpoint of anti-oketsu effects, nor did they compare the activity on the mesenteric artery with that on the aorta. As far as we know, this is the first work that compares the vasorelaxant activities of different compounds on rat aorta and mesenteric artery.

Thus, we demonstrated that the comparative study of various blood vessels, such as aorta and mesenteric artery, is important for the evaluation of the anti-oketsu effects of various compounds. The fact that natural compounds that show different mechanisms of action on the two blood vessels exist underscores the need to conduct such a study.

Although the mechanisms underlying the impairment of peripheral blood circulation in the oketsu syndrome remain unclear, our findings not only support the traditional use of Sappan Lignum but also offer a credible explanation for its beneficial effects on the vascular system.

\section{REFERENCES}

1) "International Collation of Traditional and Folk Medicine," Vol. 3, ed. by Chung K. S., World Scientific Publishing, Singapore, 1998.

2) "The Encyclopedia of Wakan-yaku," Vol. 2, ed. by Namba T., Hoikusha, Tokyo, 1991.

3) Liu A. L., Shu S. H., Qin H. L., Lee S. M., Wang Y. T., Du G. H.,
Planta Med., 75, 337-339 (2009).

4) Yodsaoue O., Cheenpracha S., Karalai C., Ponglimanont C., Tewtrakul S., Phytother. Res., 23, 1028-1031 (2009).

5) Shen J., Zhang H., Lin H., Su H., Xing D., Du L., Eur. J. Pharmacol., 558, 88-95 (2006)

6) Washiyama M., Sasaki Y., Hosokawa T., Nagumo S., Biol. Pharm. Bull., 32, 941—944 (2009).

7) Xie Y. W., Ming D. S., Xu H. X., Dong H., But P. P. H., Life Sci., 67, 1913-1918 (2000)

8) Hu C. M., Kang J. J., Lee C. C., Li C. H., Liao J. W., Cheng Y. W., Eur. J. Pharmacol., 468, 37-45 (2003).

9) Matsumoto T., Kobayashi T., Kamata T., Curr. Cardiol. Rev., 2, 185191 (2006).

10) Busse R., Edwards G., Félétou M., Fleming I., Vanhoutte P. M., Weston A. H., Trends Pharmacol. Sci., 23, 374-380 (2002).

11) Shimokawa H., Yasutake H., Fujii K., Owada M. K., Nakaike R., Fukumoto Y., Takayanagi T., Nagao T., Egashira K., Fujishima M., Takeshita A., J. Cardiovasc. Pharmacol., 28, 703-711 (1996).

12) Nagai M., Nagumo S., Eguchi I., Lee S. M., Suzuki T., Yakugaku Zasshi, 104, 935-938 (1984).

13) Nagai M., Nagumo S., Lee S. M., Eguchi I., Kawai K., Chem. Pharm. Bull., 34, 1-6 (1986).

14) Nagai M., Nagumo S., Heterocycles, 24, 601-605 (1986).

15) Nagai M., Nagumo S., Chem. Pharm. Bull., 35, 3002-3005 (1987).

16) Kasai H., Kobayashi N., Sasaki Y., Nagumo S., Nagai M., Abstract of Papers, the 52nd Annual Meeting of the Japanese Society of Pharmacognosy, 2005, p. 162.

17) Nagai M., Nagumo S., Chem. Pharm. Bull., 38, 1490-1494 (1990).

18) Matsumoto T., Kobayashi T., Kamata T., Am. J. Physiol. Heart Circ. Physiol., 285, H283-H291 (2003).

19) Sasaki Y., Hosokawa T., Nagai M., Nagumo S., Biol. Pharm. Bull., 30, 193-196 (2007)

20) Chiou W. F., Yen M. H., Chen C. F., Eur. J. Pharmacol., 204, 35-40 (1991).

21) Chiou W. F., Chou C. J., Shum A. Y. C., Chen C. F., Eur. J. Pharmacol., 215, 277-283 (1992). 